\title{
Microflora microbiological characteristics of saline soils
}

\author{
Igor Prikhodko ${ }^{1, *}$, Artyom Verbitsky ${ }^{1}$, Stanislav Vladimirov ${ }^{1}$ and Tatyana Safronova $^{1}$ \\ ${ }^{1}$ Kuban State Agrarian University named after I. T. Trubilin, 13, Kalinina str., 350044, Krasnodar, \\ Russia
}

\begin{abstract}
The purpose of current work was isolation of halophilic bacteria from saline lands in some regions of the Krasnodar Territory, after mycological analysis, there followed the study and characterization of microorganisms with further creation on effective biocomplex basis, contributing to restoration of arid and saline irrigated areas. Soil samples were taken from saline lands of the Krasnodar Territory, the salt composition of which was studied in the work process. There were determined total titer of microorganisms and the titer of nitrogen-fixing bacteria on the corresponding selective media in the samples. Twenty-five pure bacterial cultures were isolated from the samples and its morphophysiological and biochemical properties, as well as its nitrogenfixing activity, were studied. As a result, seven cultures with relatively high nitrogen-fixing activity were selected, as well as resistance to various environmental factors, particularly, to high concentrations of $\mathrm{NaCl}, \mathrm{pH}$ alkaline values and to sharp temperature drops. After appropriate phylogenetic analysis, selected crops may become a potential target for creating a complex biological fertilizer, contributing to the restoration of dry and saline lands and increase their fertility.
\end{abstract}

\section{Introduction}

Land degradation under the influence of salts is a serious threat to Russian agriculture. The existing problem is relevant for both arid and semi-arid regions. The main causes of the problem are intensification of production, reduction of areas with high natural agroresource potential, growth in demand for agricultural products, quality deterioration and irrigation water quantity reduction, lack of proper attention to the methods for producing agricultural products: crop rotation failure, irrigation regimes violation, failure to comply with all regulated autumn-spring field work $[1,2]$. All this, along with lack of the required amount of precipitation and high average monthly temperatures, will inevitably lead not only to salinization of the arable layer, but also to secondary salinization processes, leading to significant deterioration of soil fertility, up to its complete withdrawal from crop rotation. It is obvious that the growth dynamics of areas exposed to degassing processes will increase, in particular, areas subject to salinization [3, 4].

Therefore, it is important to understand the processes occurring in saline soils,

*Corresponding author: prihodkoigor2012@yandex.ru 
especially those, that are involved in the nutrient cycle. Thus, the effects of excess salt content in the soil solution include a decrease in water absorption due to low osmotic potential, high $\mathrm{pH}$ and ionic competition, which limits absorption of nutrients, which not only reduce plant growth, but also have negatively affect on growth and activity of soil microbial biomass, and also adversely affect the course of biochemical processes necessary to maintain the organic matter of the soil $[5,6]$. Reclamation of saline arable land is very relevant for Russia as a whole and for the Kuban in particular, since at present the damage caused by salinization is increasing.

These problems can be solved with biofertilizers, as some microbes respond to low osmotic potential, accumulating osmolytes to retain water, while sensitive microbes die [7, 8]. Using live bacteria (biofertilizers) accelerates mineralization of organic residues in the soil, thereby making nutrients more affordable. At the same time, due to exposure to living bacteria from biocomplex fertilizer, the absorption of heavy metals is reduced $[9,10]$. Bacterial-based biological fertilizer uses the metabolic activity of living microorganisms to convert raw materials, such as atmospheric nitrogen and phosphorus and potassium minerals, into plant nutrients, and the conversion and release of such nutrients by microorganisms is partially controlled by nutrient content in the soil.

Considering the above, the main goal of current work is: study of soil biota (fungi, bacteria), isolation of $(\mathrm{N})$ fixing microorganisms from saline lands of the Krasnodar Territory, study of its characteristics, followed by the use of salt tolerant microorganisms as bio-fertilizers in saline soil.

\section{Materials and methods}

Evaluation of bacterial and fungal soil microflora from sampling sites.

Research methods: sampling from plots was carried out from a depth of $0-20 \mathrm{~cm}$. Mycological soil analysis (structure and quantity assessment of soil fungi) conducted according to the Russian generally accepted standard method. The number of ammonifiers, nitrifiers and denitrifiers was determined by the methods described in the Russian standard «Organic fertilizers». Microbiological analysis methods». For mycological analysis, Chapek's nutrient medium was used [11]. The temperature of fungi germination in thermostat was $25{ }^{\circ} \mathrm{C}$. Repetition - sevenfold [12]. Quantitative analysis of microorganisms was carried out on the seventh day by counting colonies in Petri dishes. Microorganisms identification was carried out at the onset of the sporulation stage in colonies [13].

The control is represented by microbial cultures of genuses: Pseudomonas, Rhizobium и Azotobacter.

Crops cultivation was carried out in a liquid nutrient medium for microbes cultivation, consisting of meat water and peptone peptone - broth and meat-peptone agar. Ashby agar medium was used as the minimum selective medium for revealing nitrogenobacter in the soil. Nitrogen-fixing microorganisms activity was determined using semi-quantitative method with (NFB) as an indicator. Анализ проводился по методике, описанной в методическом руководстве [14]. The method is based on the fact that during microorganism growth the ammonia is released and stains the indicator in different shades of blue, the degree of nitrogen-fixing activity of the tested cultures is estimated by the indicator's color intensity. Microbial cultures were stained according to the Gram method, stained according to the method described in the methodological manual of the standard method [15]. 


\section{Results and discussion}

Mycological analysis of the soil. The analysis results.

Mycological analysis of the soil revealed fungi of the next genuses (Fig. 1): 1 . Alternaria 2. Aspergillus 3. Cladosporium 4. Fusarium 5. Mucor 6. Penicillium 7. Rhizopus 8. Trichoderma 9. Verticillium

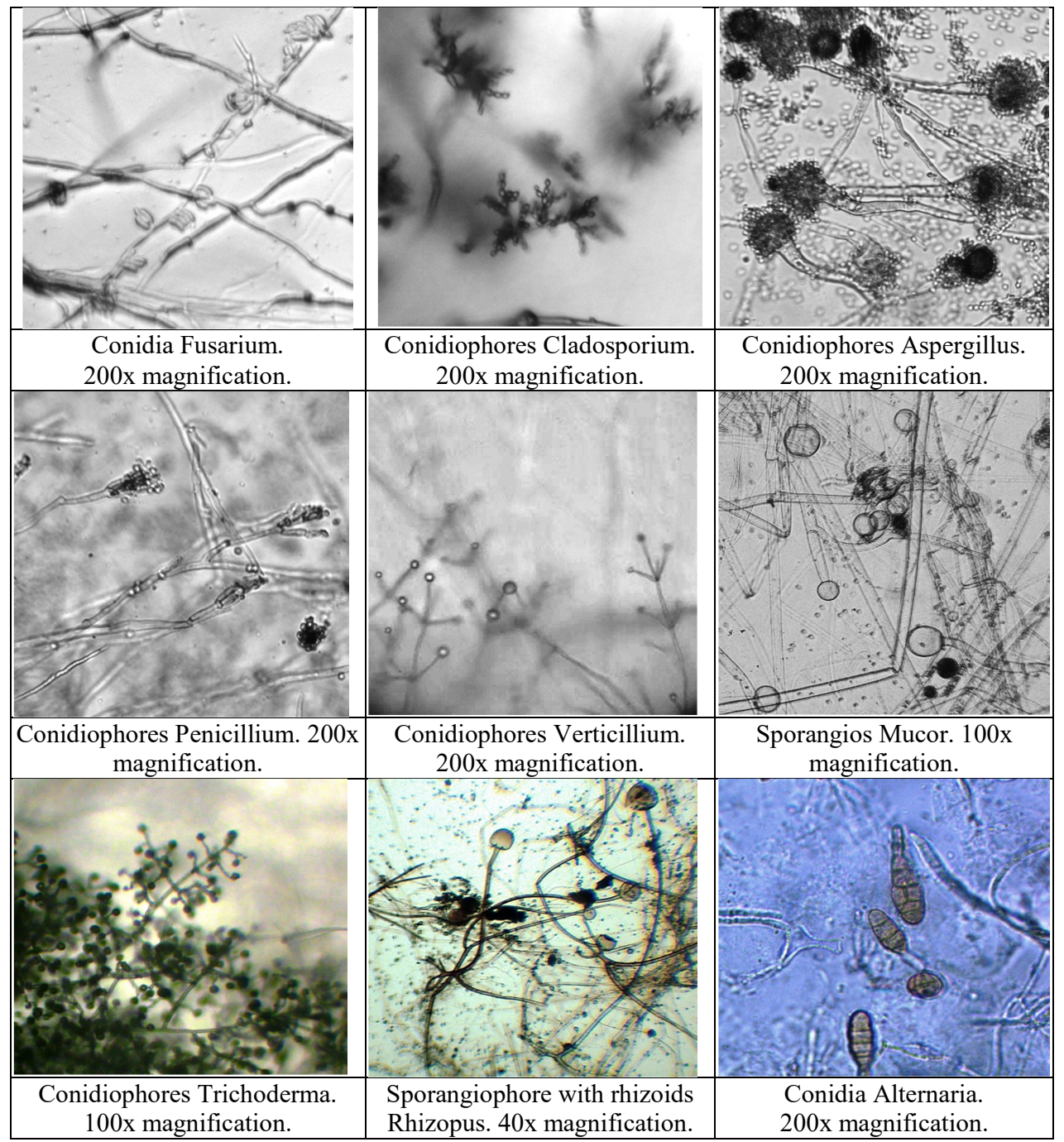

Fig. 1. Morphological characteristics of microorganisms, detected by microscopy of colonies, formed when sowing on Chapek's nutrient medium extracts of the studied soil samples.

Five representatives of wich - Alternaria, Fusarium, Verticillium, Rhizopus, Cladosporium are pathogens of cultivated plants, Aspergillus, Penicillium - conditional suppressors; Trichoderma - suppressor.

Fungi of the genus Alternaria - agricultural crop pathogens. Vegetable and fruit crops cause the most damage, causing spotting on plant leaves. The reservoir for fungi is the plant debris from which they enter the soil. Alternaria was found in small amounts in soil sample 649-3, and singly in 651-3 (table 1). 
Table 1. The micromycete complex structure in soil samples.

\begin{tabular}{|c|c|c|c|c|c|c|c|c|c|c|c|}
\hline \multirow[b]{2}{*}{ 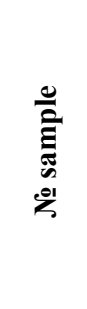 } & \multicolumn{11}{|c|}{ The content of mushroom propagules in $1 \mathrm{~g}$ of absolutely dry soil } \\
\hline & 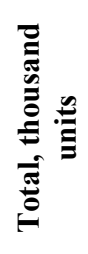 & 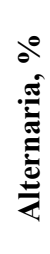 & 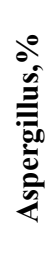 & 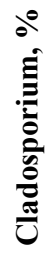 & 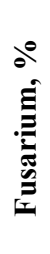 & $\begin{array}{l}\stackrel{0}{0} \\
\dot{\bar{\Xi}} \\
\stackrel{\Xi}{\Sigma}\end{array}$ & 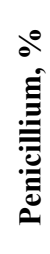 & 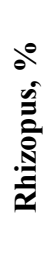 & 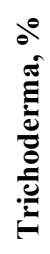 & 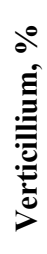 & $\begin{array}{l}\stackrel{0}{0} \\
\stackrel{0}{0} \\
\stackrel{0}{0}\end{array}$ \\
\hline $648-3$ & 44.6 & - & 1 & 19 & 27 & 1 & 25 & 7 & - & - & 19 \\
\hline $649-3$ & 13.7 & 7 & 22 & 2 & 29 & 1 & 21 & 3 & - & - & 15 \\
\hline $650-3$ & 65.4 & - & 1 & - & 18 & 2 & 76 & 2 & - & - & 1 \\
\hline $651-3$ & 25.0 & 1 & 4 & - & 27 & 3 & 7 & 3 & 6 & 9 & 40 \\
\hline $652-3$ & 53.3 & - & 9 & 11 & 17 & 1 & 57 & 1 & 2 & 2 & - \\
\hline
\end{tabular}

Most fungi of the genus Fusarium - wide specialized pathogens, able to damage almost all cultivated plants, causing root rot, fusarium leaf burn. The source of infection is plant debris affected by the pathogen, soil and seed, where fungi can persist for a long time. Fungi of this genus develop mainly in heavy and waterlogged soils.

In all studied samples, fungi of this genus are represented in amounts significantly exceeding threshold content of Fusarium fungi infectious structures (1000 pcs / g of dry soil).

In samples 651-3 и 652-3 the presence of fungi of the genus Verticillium, individual representatives of wich cause wilting (wilt) or drying out of plants.

Rhizopus, Mucor and Cladosporium may cause moldy seeds, reducing germination. Fungi of the genus Cladosporium may also be vegetable pathogens. Rhizopus and Mucor were found in all samples in small quantities, not harmful to plants. Cladosporium represented mainly in samples 648-3 and 652-3.

Of the saprotrophic fungi that have suppressive properties against identified pathogens, representatives of the genus Penicillium predominated Content of Aspergillus in the studied samples was lower, than of Penicillium genus representatives, except sample 649 - 3.

Excess amount Penicillium and Aspergillus in the soil may pose a certain danger to plants because of its ability to produce phytotoxins. The identified microorganisms diversity in the samples, and species composition of fungi of the genus Penicillium indicate low probability of phytotoxins accumulation in the soil associated with fungal microflora activity.

In samples 651-3 and 652-3 there is a small amount of Trichoderma, being a good suppressor in the soil.

When comparing the samples, it can be seen that the most atypical microorganisms' composition is in the samples $649-3$ and $651-3$, where total number of microorganisms is two times lower than other options, and low content of Penicillium is also noted in sample 651-3 (Fig. 2). This may indicate unfavorable soil conditions for microflora formation in comparison with soils from other samples. 


\section{INTERAGROMASH 2020}

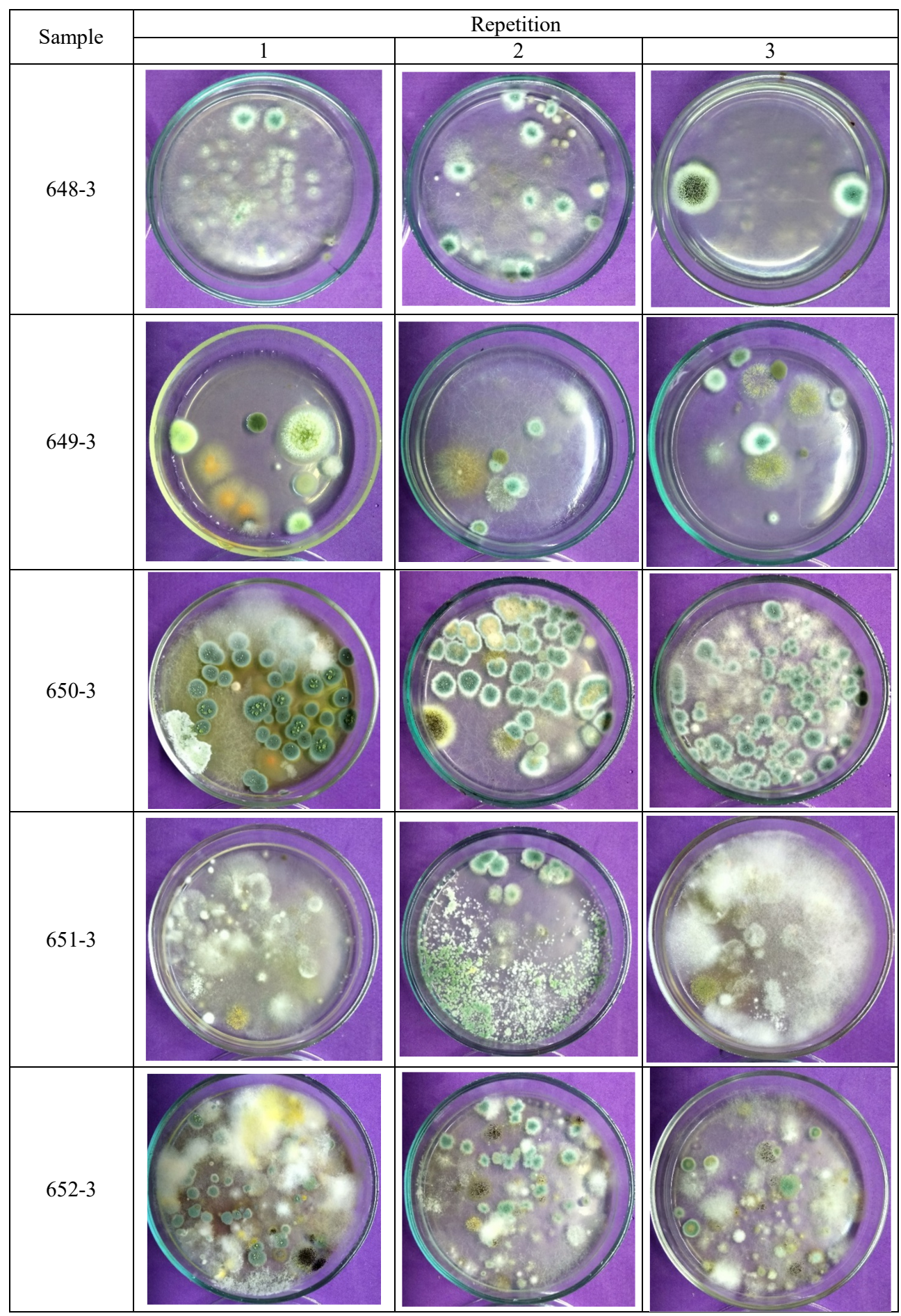

Fig. 2. Colonies of microorganisms identified by mycological analysis of the soil.

In the analyzed soil sample there were revealed phytopathogens of the next genus Fusarium, Verticillium and Cladosporium (Table 2); (Fig. 3). All pathogens are present in 
the sample in small quantities. Fungi of the genus Fusarium slightly exceeded the permissible norm of 1000 propagules per gram of dry soil.

Table 2. The structure of the micromycete complex in soil samples.

\begin{tabular}{|c|c|c|c|c|c|c|c|}
\hline \multicolumn{8}{|c|}{ The content of mushroom propagules in 1 gram of dry soil, thousand units } \\
\hline \multirow{2}{*}{ 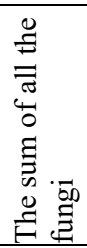 } & \multicolumn{3}{|c|}{ Phytopathogens } & \multicolumn{3}{|c|}{ Suppressors } & \multirow{2}{*}{ 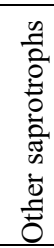 } \\
\hline & 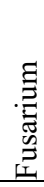 & 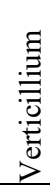 & 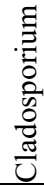 & 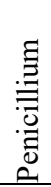 & 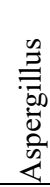 & 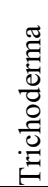 & \\
\hline 60 & 3 & 2 & 2 & 3 & 20 & 1 & 28 \\
\hline
\end{tabular}

Despite a small number of phytopathogens, gradually, its number may increase significantly, since suppressive microflora (fungi of genus Penicillium, Aspergillus and Trichoderma) in the soil sample is also insufficiently represented, which does not allow it to effectively restrain the development of identified phytopathogens.

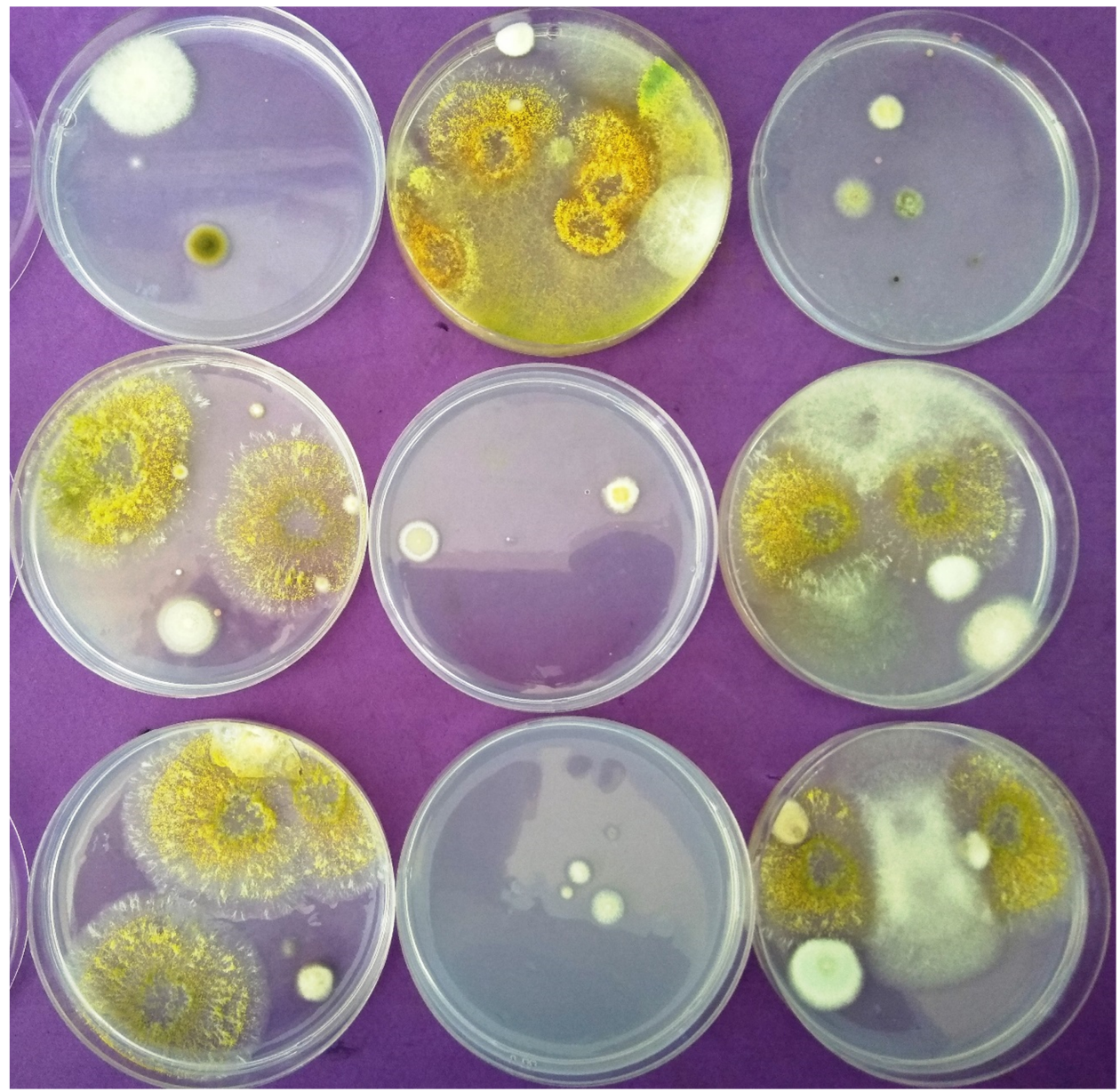

Fig. 3. Petri dishes with fungal colonies grown on culture medium after inoculation of extracts from the soil sample.

The studied samples varied in quantity (Table 3) and properties (Fig. 4) of fungi genus 
Penicillium, which made up the dominant group in the micromycete complex.

Table 3. The structure of the micromycete complex of the upper soil layer.

\begin{tabular}{|c|c|c|c|c|c|c|}
\hline \multirow{3}{*}{$\begin{array}{c}\text { Soil } \\
\text { characteristics }\end{array}$} & \multicolumn{6}{|c|}{ The number of soil fungi in one gram of dry soil, thousand units } \\
\hline & \multirow{2}{*}{$\begin{array}{c}\text { The sum } \\
\text { of all } \\
\text { fungi }\end{array}$} & \multirow{2}{*}{\begin{tabular}{|c|} 
Phytopathogens \\
Rhizopus \\
\end{tabular}} & \multicolumn{3}{|c|}{ Suppressors } & \multirow{2}{*}{$\begin{array}{c}\text { Other } \\
\text { saprotrophs }\end{array}$} \\
\hline & & & Penicillium & Aspergillus & Trichoderma & \\
\hline Weak salted & 202 & 6 & 128 & 2 & 22 & 44 \\
\hline Saline & 159 & - & 90 & 3 & 22 & 44 \\
\hline Highly salted & 106 & - & 38 & 6 & 16 & 46 \\
\hline
\end{tabular}

Fig. 4 shows that from the sample from weak salinity medium, while plating on medium there grew a large number of penicillium colonies with red pigment - toxin released by the fungus into the environment. In the medium where salinity of penicillium colonies of this kind is less, and with strong salinity, such colonies were absent. Large number of penicillas with red pigment is a sign of soil "fatigue".

All studied soil samples were characterized by good suppression, since fungi predominated in micromycetes structure. - suppressors: Penicillium, Aspergillus, Trichoderma, moreover, the proportion of trichoderma was significant.

Pathogens, causing root rot (Fusarium, Pythium etc.) were not identified.

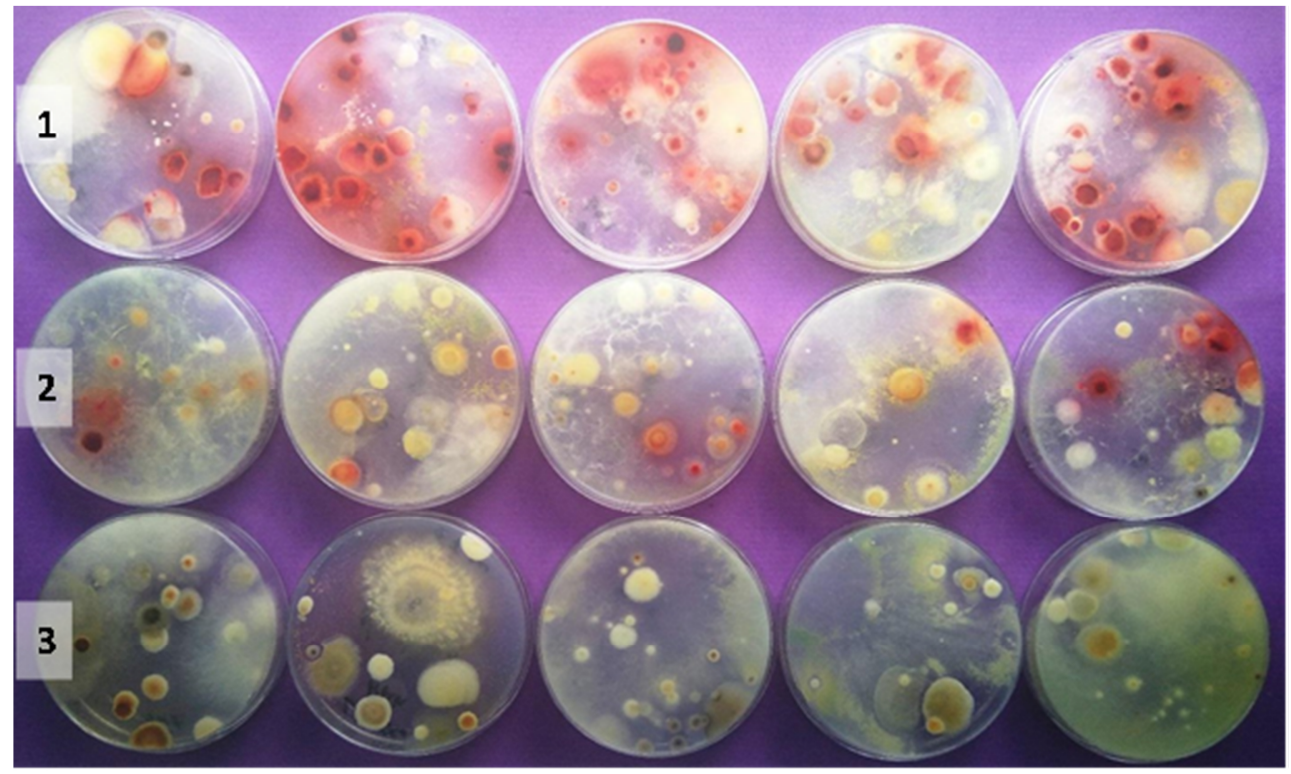

Fig. 4. Colonies of soil fungi grown in Petri dishes at Chapek's nutrient medium, after sowing soil samples. Underside of Petri dish. Explanation of symbols: 1 - weak salted, 2 - salted, 3 - highly salted.

Ammonifiers:

The concentration of ammonifiers in soil samples, determined by dilution on medium GRM №1, made up (CFU/g of dry soil (Fig. 5)): 1. «weak salted» $3 * 10^{7} ; 2$. «salted»$6^{*} 10^{6} ; 3$. «highly salted» $-6^{*} 10^{6}$. 


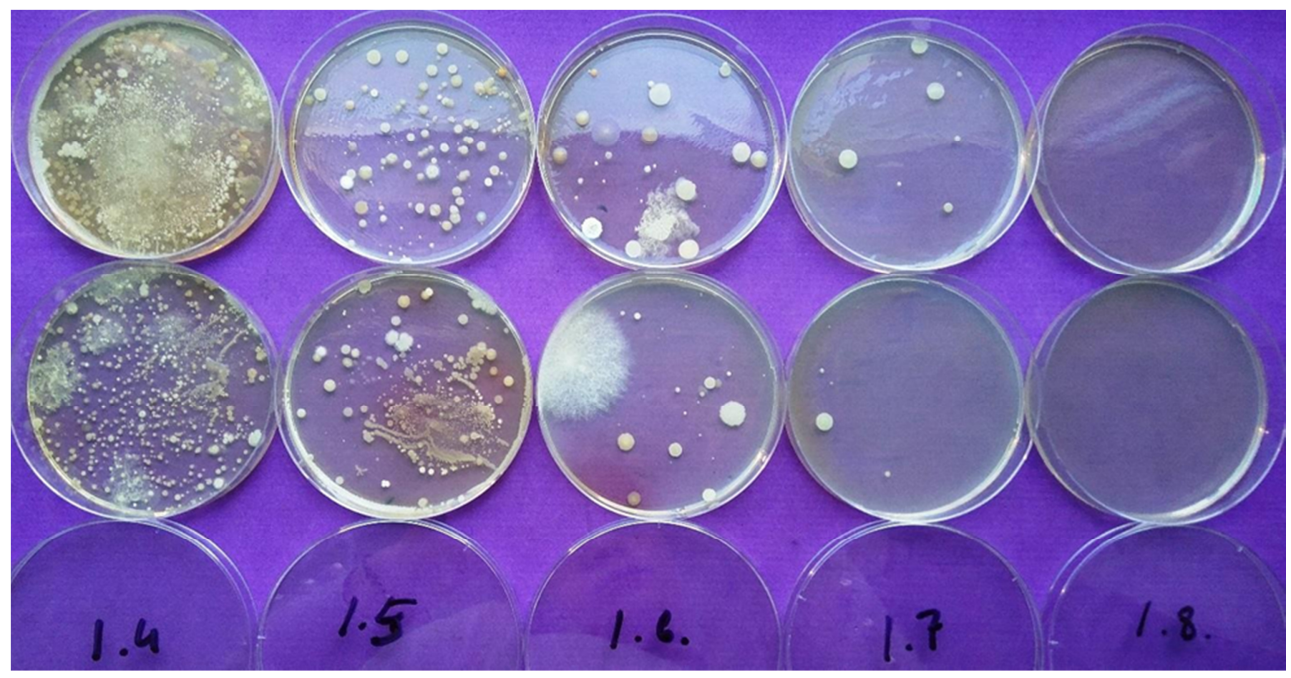

Fig. 5. The obtained series of dilutions in determining the ammonifiers concentration in the soil sample. Option "weak salted".

Nitrificators:

The nitrification activity of microorganisms in all soil samples was low and was not detected by the method, recommended by Russian standards.

Denitrifiers:

The concentration of denitrifiers in soil samples made up (CFU/g of dry soil (Fig. 6)): 1 . «weak salted $1 * 10^{5} ; 2$. «salted» $-1 * 10^{6} ; 3$. «highly salted» $-1 * 10^{4}$.

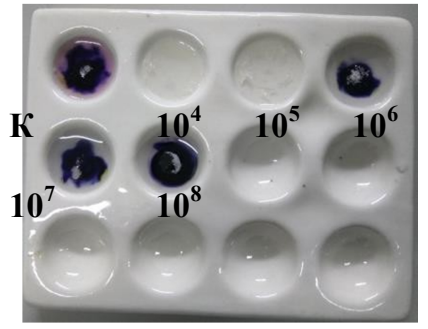

«weak salted»

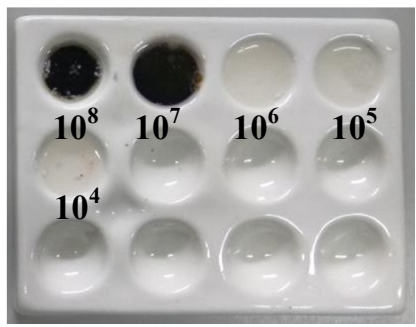

«salted»

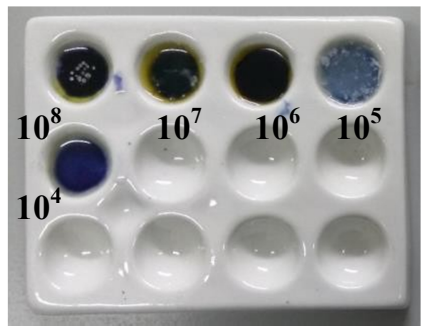

«highly salted»

Fig. 6. The analysis results for nitrates presence in the Beryozova nutrient medium, in which the soil samples were sown. Dark blue color - nitrates are present in the medium, transparent - nitrates processed by denitrifiers.

\section{Conclusions}

According to the soil microorganisms' studies results with varying degrees of salinization, seven nitrogen-fixing strains were isolated from mycological samples, which due to its properties, such as resistance to high temperature extremes, ability to develop in $\mathrm{pH}$ highvalue environments, can be recommended for the development of highly effective multipurpose fertilizer.

Fertilizers of this kind, obtained taking into account its species affiliation by phylogenetic analysis of the obtained strains, are universal in nature and optimally suitable for use not only in arid and semi-arid zones, but throughout Russia.

The use of such complex biological fertilizers will allow not only to preserve the agro- 
resource state of soils, but also to improve land reclamation indicators, increase productivity and reduce the cost of agricultural production.

\section{References}

1. Chen Shibao, Wang Duo, Wang Meng, Chen Li, Zhao Chunmei, Chemosphere 236, 124372 (2019)

2. S.Donald Lawrence, M. Mohammad, Critical Reviews in Biotechnology 39(8), 9991014 (2019)

3. R. Behnam, E. Ali, Khoshkholgh-Sima Nayer Azam, R. Narges, Journal of Environmental Management 250, 109476 (2019)

4. Zhang Fenghua, Wang Dan, Wang Weichao, Yang Lei, Zhao Yaguang, Archives of Microbiology 201(9), 1219-1232 (2019)

5. Chen Shibao, Wang Duo, Wang Meng, Chen Li, Ecotoxicology and Environmental Safety 182, 109431 (2019)

6. Shen Qirong, Li Rong, Long Xiaohua, Long Xiaohua, Cai Feng, Science of the Total Environment 687, 601-609 (2019)

7. T. Safronova, O. Degtyareva, S. Vladimirov, I. Prikhodko, Research Journal of Pharmaceutical, Biological and Chemical Sciences 9(6), 1845-1852 (2018)

8. Chen Shibao, Wang Duo, Wang Meng, Chen Li, Environmental Pollution 252, 16091621 (2019)

9. E. Kuznetsov, T. Safronova, I. Sokolova, A. Khadzhidi, A. Gumbarov, JEMT 81(17), 78-83 (2017)

10. S. Muhammad Rizwan, A. Muhammad Sohail, N. Muhammad Asif, A. Sajjad, T. Muhammad Usman, Ecotoxicology and Environmental Safety 178, 33-42 (2019)

11. S. Vladimirov, I. Prikhodko, A. Verbitskiy, Journal of Agriculture and Environment 1(9), 15 (2019)

12. M. Lopez-Fernandez, A. Cherkouk, S. Kluge, M. Bachran, Microbial Ecology 78(2), 494-505 (2019)

13. O. Degtyareva, T. Safronova, I. Rudchenko, I. Prikhodko, IOP Conf. Series: Materials Science and Engineering 698, 022015 (2019) doi:10.1088/1757-899X/698/2/022015

14. Ashbolt Nicholas J., Tang Jingchun, Liu Xiaomei, Zhen M., Liu Qinglong, Song Benru, Journal of Environmental Sciences (China), 81, 80-92 (2019)

15. Dong Hailiang, He Zhili, Deng Ye, M.P.J. Virta, Feng Kai, Hou Weiguo, Wang Shang, Qin Huayu, Microbiome 7(1), 71 (2019)

16. Sa Tongmin, Kim Kiyoon, Krishnamoorthy Ramasamy, Samaddar Sandipan, Chatterjee Poulami, Jeon Sunyoung, Applied Soil Ecology 137, 96-105 (2019)

17. Hou Zhen'an, Min Wei, Guo Huijuan, Hu Zhiqiang, Zhang Huimin, Water, Air, and Soil Pollution 230(2), 44 (2019) 